\title{
Reductions in calcitonin gene-related peptide may be associated with the impairment of the contralateral testis in unilateral cryptorchidism
}

\author{
BAOPING ZHU, QING LIU, LI LIN and XINMIN ZHENG \\ Department of Urology, Zhongnan Hospital of Wuhan University, Wuhan, Hubei 430071, P.R. China
}

Received May 6, 2014; Accepted December 23, 2014

DOI: $10.3892 /$ etm.2015.2343

\begin{abstract}
The aim of the present study was to investigate the mechanism underlying the impairment of the contralateral testis in unilateral cryptorchidism in experimental rats using a molecular neurophysiological approach. Thirty-six male rats (21 days old) were divided into a cryptorchidism group, a cryptorchidism with division of the genitofemoral nerve (GFN) group and a control group ( $n=12 /$ group). The distribution of the calcitonin gene-related peptide (CGRP) immunoreactive nerve fibers in the testes was studied using an immunohistochemistry technique. Germ cell apoptosis was detected using the terminal deoxynucleotidyl-transferase-mediated dUTP nick end labeling method. The concentration of malondialdehyde (MDA) in the testis tissue was evaluated using a spectrophotometric determination method, and the ultrastructure of Sertoli cells was observed using transmission electron microscopy. It was found that, 100 days after the surgery, the concentration of CGRP in the cryptorchidism group was decreased significantly, whereas the levels of MDA and the number of apoptotic germ cells were increased significantly compared with the control group $(\mathrm{P}<0.01)$. Following the division of the GFN, the damaging effects were decreased $(\mathrm{P}<0.01)$. The impairment mechanism may therefore be associated with a reduction in the level of CGRP in the contralateral testis. The reflex decrease in CGRP may be caused by germ cell apoptosis, decreased blood flow and oxygen levels, and the increase in reactive oxygen free radicals and lipid peroxidation.
\end{abstract}

\section{Introduction}

Cryptorchidism is a common congenital abnormality with high incidence and prevalence (1). The prevalence varies with apparent geographical differences (2). In general, the

Correspondence to: Dr Baoping Zhu, Department of Urology, Zhongnan Hospital of Wuhan University, 169 Donhu Road, Wuhan, Hubei 430071, P.R. China

E-mail: baopingzhuz@126.com

Key words: cryptorchidism, calcitonin gene-related peptide, genitofemoral nerve, apoptosis incidence rate is $30.3 \%$ for preterm infants, $4 \%$ for newborns and $0.8 \%$ for one-year-old babies. The condition also represents one of the major causes of male infertility. The infertility rate of patients with bilateral cryptorchidism may be as high as $50-100 \%$, whereas the infertility rate of patients with unilateral cryptorchidism is $30-60 \%$ (3). The reason for this may be that the incidence rate of patients with bilateral cryptorchidism is very low, and with the development of society, the majority of people select the surgery in childhood (4). The dysfunction of the contralateral testis could be an important cause of male infertility with unilateral cryptorchidism symptoms (4); however, the mechanism associated with the damage has not been fully elucidated. Foresta et al (5) proposed that unilateral cryptorchidism is a congenital disease and that the damage to the contralateral testicle is the ultimate result of this inherent disease. Vasquez et al (6) demonstrated that the concentrations of follicle stimulating hormone, luteinizing hormone and testosterone were associated with the sperm density of patients with unilateral cryptorchidism, and that damage to the contralateral testicular was caused by endocrine abnormalities. It is additionally believed that the blood-testis barrier in unilateral cryptorchidism could be the cause of autoimmune reactions or allergic orchitis, which could then cause contralateral testis damage $(7,8)$.

The genitofemoral nerve (GFN) originates in the first and second lumbar plexus, cycles through the psoas muscle and then forms the reproductive and groin branches. The reproductive branch is a sensory-motor hybrid neuron that enters the inguinal canal at the inner ring and descends along the inguinal canal. The fibers are widely distributed in the cremaster muscle, gubernaculum and testicular hydrocele (9). By contrast, the groin branch enters the testicular vascular system directly and is the most important afferent nerve supplying the testis (10). In the present study, the nerve distribution of the contralateral testis, including the areas of calcitonin gene-related peptide (CGRP)-positive cells and certain associated factors, was observed, and the mechanism underlying the impairment of the contralateral testes in unilateral cryptorchidism was explored in experimental rats.

\section{Materials and methods}

Establishment of the experimental model. Thirty-six male Sprague Dawley rats (weight, 120-180 g) were randomly 
assigned to the control (group A), left unilateral cryptorchidism (group B) and left unilateral cryptorchidism with division of the left GFN (group C) groups ( $\mathrm{n}=12$ /group) A ventral midline incision was performed in group $\mathrm{C}$ following anesthesia and the GFN was separated from the leading edge of the left psoas muscle. The ipsilateral testicular gubernaculum was cut off, the testis was fixed to the posterior abdominal wall with 4-0 sutures (without damage) and the abdomen was closed in two layers. The rats from group B were treated in the same way but the GFN was kept intact. The operation was finished following the opening of the abdominal wall of the rats. For the rats in group A, no additional treatment was applied following the closure of the abdominal wall and the rats were raised as normal. All rats were sacrificed by rapid cervical dislocation subsequent to a further 100 days of feeding, and the contralateral testis from each rat was collected for further measurement. The weights of the testes were determined. The housing of the rats and the procedures involving the experimental animals were in accordance with the Guide for the Care and Use of Laboratory Animals (eighth edition, 2011). All animal experiments were approved by the Animal Care and Use Committee of Wuhan University (Wuhan, China).

Immunohistochemistry to analyze the CGRP-positive cells of the contralateral testis. Conventional specimen slices were prepared and the immunohistochemical staining method was applied. Following deparaffinization and dehydration, the sections underwent $0.5 \%$ potassium citrate (Wuhan Boster Biological Technology Co., Ltd., Wuhan, China) antigen retrieval at $100^{\circ} \mathrm{C}$ for $15 \mathrm{~min}$. The sections were then blocked with $5-10 \%$ goat serum and incubated at $37^{\circ} \mathrm{C}$ for $20 \mathrm{~min}$. The primary antibody (rabbit polyclonal anti-CGRP; cat. no. BA0204; Wuhan Boster Biological Technology Co., Ltd.) was subsequently added at a dilution of 1:100 and the samples were incubated at $37^{\circ} \mathrm{C}$ for a further $15 \mathrm{~h}$. The samples were then washed with phosphate-buffered saline (PBS) for 2 min for a total of three times, incubated with secondary antibody (biotin-labeled goat anti-rabbit immunoglobulin G; Beijing Zhongshan Biotechnology Co., Ltd., Beijing, China) at $37^{\circ} \mathrm{C}$ in a water bath for $2 \mathrm{~h}$ and re-washed with PBS for $2 \mathrm{~min}$ for a total of 3 times. Following treatment with 3,3'-diaminobenzidine solution, the sections were flushed, counterstained with hematoxylin, washed with water, dehydrated, cleared, mounted on slides and observed under the microscope. Ten immunohistochemical staining slices were randomly selected from each group and the average luminosity, area and positive rate of CGRP-positive cells were detected with an automatic image analyzer (HPIAS-2000 image analysis software; Tongji Qiangping Image Engineering Co., Wuhan, China).

Determination of malondialdehyde (MDA) content in samples. The MDA content in the samples was determined using a chemical colorimetric method and an MDA assay kit (Nanjing Jiancheng Institute of Biology, Nanjing, China). The assay was performed in accordance with the manufacturer's instructions.

Detection of apoptosis. Testicular germ cell apoptosis was detected using the terminal deoxynucleotidyl-transferase-mediated dUTP nick end labeling method and an apoptosis detection

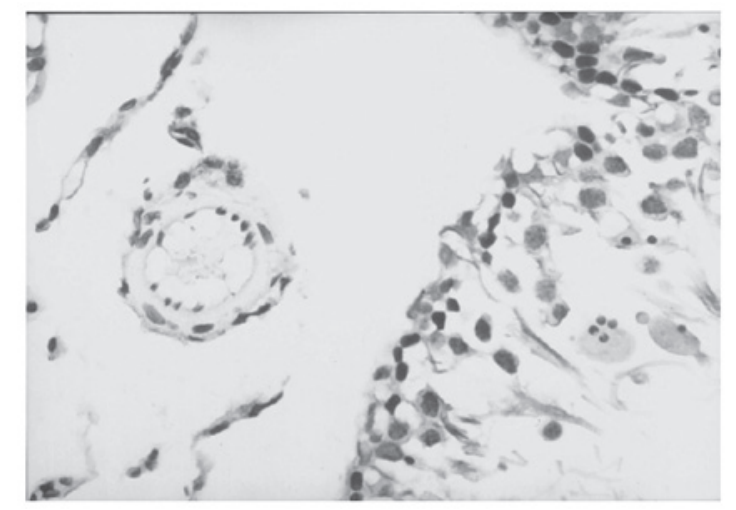

Figure 1. Testicular immunohistochemical staining of group A. Calcitonin gene-related peptide-positive fibers are distributed along the blood vessels (3,3'-diaminobenzidine; magnification, x400).

kit (Wuhan Boster Biological Technology Co., Ltd. in accordance with the manufacturer's instructions. Thirty seminiferous tubule sections were selected from each slice and the percentage of positive cells was calculated to give the apoptosis index (AI).

Ultrastructural changes of testicular Sertoli cells. The ultrastructural changes of testicular Sertoli cells were observed using transmission electron microscopy (TEM; JEM100CXII; JEOL Ltd., Beijing, China).

Statistical analysis. Values are presented as the mean \pm standard deviation. All data analysis was performed with SPSS 17.0 statistical software (SPSS, Inc., Chicago, IL, USA). The data were analyzed using the Student's t-test for comparisons among groups. $\mathrm{P}<0.05$ was considered to indicate a statistically significant difference.

\section{Results}

Weight, AI and MDA. Compared with the results for group A, the weight of the contralateral testis in group B was significantly reduced and the AI and levels of MDA were significantly increased $(\mathrm{P}<0.01)$. Compared with the results for group $\mathrm{B}$, the weight of the contralateral testis in group $\mathrm{C}$ was significantly increased. The MDA levels and AI in group $\mathrm{C}$ were significantly reduced compared with those in group $\mathrm{B}(\mathrm{P}<0.01)$, but remained higher than those in group $\mathrm{A}(\mathrm{P}<0.05)$ (Table I).

Immunohistochemistry. Immunohistochemistry showed that there were numerous nerve fibers rich in CGRP-positive cells in the interstitial tissue of the testis in group A. The brown immunoreactive substances in the cells exhibited a granular distribution. The staining also revealed intensive agglomeration, with positive fibers distributed along the perivascular nerves belonging to the peripheral component of the sympathetic nervous system. The vascular smooth muscle was thick and lined by endothelial cells (Fig 1). By contrast, the seminiferous epithelium in group B was thin and the number of CGRP-positive cells was significantly reduced $(\mathrm{P}<0.01)$. In addition, the vascular wall was thin and the diameter was narrow. The neural image analysis results of the CGRP-positive cells are shown in Table II. Compared with group A, the CGRP-positive nerve distribution and content in 
Table I. Changes in the weight, MDA levels and AI of the contralateral testis in groups A, B and C.

\begin{tabular}{llrr}
\hline Group & Weight $(\mathrm{mg})$ & MDA $(\mathrm{nmol} / \mathrm{mg})$ & AI $(\%)$ \\
\hline A & $746.33 \pm 95.22$ & $2.41 \pm 0.50$ & $6.48 \pm 1.64$ \\
B & $504.71 \pm 50.10^{\mathrm{a}}$ & $7.24 \pm 1.19^{\mathrm{a}}$ & $17.24 \pm 3.36^{\mathrm{a}}$ \\
C & $642.28 \pm 69.51^{\mathrm{b}}$ & $3.63 \pm 0.72^{\mathrm{b}}$ & $8.06 \pm 2.07^{\mathrm{b}}$ \\
\hline
\end{tabular}

${ }^{\mathrm{P}}<0.01$ and ${ }^{\mathrm{b}} \mathrm{P}<0.05$ versus group $\mathrm{A}$. Group $\mathrm{A}$, control; group B, left unilateral cryptorchidism; group C, left unilateral cryptorchidism with division of the left genitofemoral nerve; MDA, malondialdehyde; AI, apoptosis index.

Table II. Neural image analysis results of the CGRP-positive cells in the contralateral testis in groups A, B and C.

\begin{tabular}{lccr}
\hline Group & Average area $\left(\mathrm{cm}^{2}\right)$ & Average luminosity (A) & Positive rate $(\%)$ \\
\hline A & $6.80 \pm 1.05$ & $0.48 \pm 0.18$ & $2.22 \pm 0.69$ \\
B & $2.11 \pm 0.65^{\mathrm{a}}$ & $0.21 \pm 0.90$ & $0.73 \pm 0.22^{\mathrm{a}}$ \\
C & $4.76 \pm 0.82$ & $0.34 \pm 0.13^{\mathrm{b}}$ & $1.49 \pm 0.38^{\mathrm{b}}$ \\
\hline
\end{tabular}

${ }^{\mathrm{a}} \mathrm{P}<0.01$ and ${ }^{\mathrm{b}} \mathrm{P}<0.05$ versus group $\mathrm{A}$. Group $\mathrm{A}$, control; group B, left unilateral cryptorchidism; group C, left unilateral cryptorchidism with division of the left genitofemoral nerve; CGRP, calcitonin gene-related peptide.

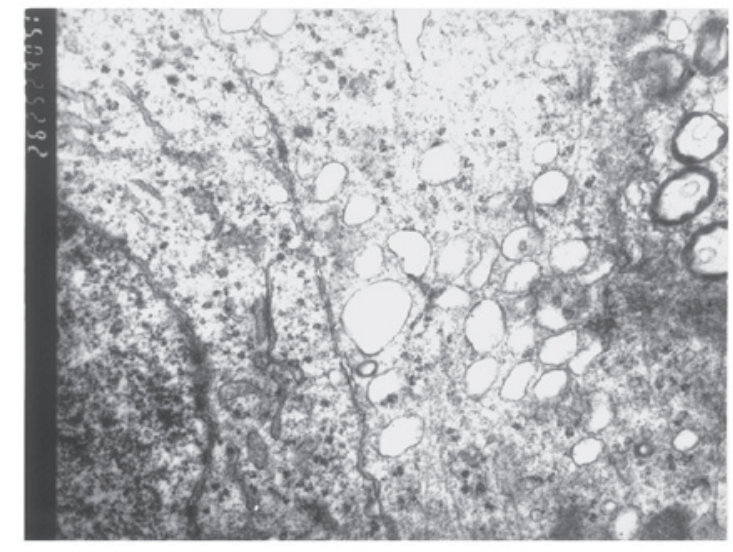

Figure 2. Testicular Sertoli cells of group B under transmission electron microscopy. Mitochondrial swelling and smooth endoplasmic reticulum dilatation are apparent (magnification, x30,000).

group B was significantly reduced $(\mathrm{P}<0.01)$; however, compared with group $\mathrm{B}$, the CGRP-positive nerve distribution and content in group $\mathrm{C}$ was significantly increased $(\mathrm{P}<0.01)$.

TEM. Observation with TEM showed that the Sertoli cells of group B were in the basement membrane of the seminiferous tubule. The cells exhibited swollen mitochondria with fewer cristae and smooth endoplasmic reticulum expansion, which suggested the existence of vacuolar changes. Additionally, the number of lysosomes was increased (Fig. 2). Few changes were observed in the Sertoli cells in groups A and C.

\section{Discussion}

Incomplete descent of the testes is the most common genital anomaly in newborn males (2). The dysfunction of the contralateral testis could be an important cause of male infertility with unilateral cryptorchidism symptoms. The present experimental results showed that the seminiferous epithelium was thinner in rats with unilateral cryptorchidism, while the MDA content was increased and there was a higher proportion of germ cells undergoing apoptosis. A positive association existed between the MDA content and the level of germ cell apoptosis. The swelling of the mitochondria and smooth endoplasmic reticulum of the Sertoli cells due to lysosomal accumulation indicated the existence of contralateral testis damage, although the potential mechanism underlying the damage is not entirely clear.

The testis and epididymis efferent nerves are highly affected by visceral sympathetic nerves, which are derived from the periarterial nerve plexuses, and both the GFN and lumbar sympathetic trunk have traffic branches. In the present study, the GFN of the cryptorchid side was removed, which reduced or prevented the degeneration of the contralateral testis and demonstrated that nerve conduction in general, and through the GFN in particular, plays an important role in the mechanism underlying contralateral testicular damage. The present results were consistent with the conclusions of Patkowski et al (11).

CGRP is a single polypeptide chain consisting of 37 amino acids that is transcribed following alternative splicing of the calcitonin gene-encoded mRNA, and is widely found in the central and peripheral nervous systems. A large number of nerve cells containing CGRP are found in the normal testis, and these exhibit sympathetic characteristics $(12,13)$. Numerous studies $(14,15)$ have shown that CGRP is the strongest neural active peptide that dilates the blood vessels of the body. Following the binding of CGRP with the corresponding target receptor, adenylate cyclase is activated to play a role similar to that of $\mathrm{Ca}^{2+}$ antagonists, by reducing the intracellular free $\mathrm{C}^{2+}$ levels and relaxing the smooth muscle of the blood vessels. CGRP can additionally antagonize the release of endothelin 
and angiotensin II by activating the cyclic adenosine monophosphate/protein kinase pathway with prostacyclin. A decrease in the concentration of CGRP can not only reduce the normal vasodilatory effect but also reduce the inhibitory effect of endothelin and angiotensin II. As a result, the vascular lumen narrows and changes occur in the hemodynamics, leading to a reduction in the perfusion of the tissue. Ischemia, hypoxia and oxygen free radicals in the cells within the tissue also increase. CGRP can additionally reduce the leakage of intracellular proteins and enzymes, promote DNA synthesis, stabilize the target organ membrane and regulate cell function by reducing $\mathrm{Ca}^{2+}$ overload, thus exerting a direct protective effect on cells (16).

MDA is a metabolite produced by the attack of oxygen free radicals on polyunsaturated fatty acids, and changes in the MDA content reflect the changes in oxygen free radical levels within the cells. An increase in MDA indicates that the intracellular oxygen free radical and lipid peroxidation levels have also increased, leading to a promotion of cell apoptosis.

The results of the present study showed that unilateral cryptorchidism could cause spermatogenic damage of the contralateral testis, and the increases in the level of germ cell apoptosis could be due to the reduction in the CGRP released by contralateral nerves. With continuous stimulation from ectopic sources, such as intra-abdominal high temperature, levels of contralateral CGRP undergo a marked reduction, as GFN fluxes to the sympathetic center; however, a decrease in the levels of CGRP can also cause a decrease in the vasodilation capacity, resulting in increased levels of intracellular oxygen free radicals and lipid peroxidation. The reduction in CGRP can simultaneously cause a further decline in cytoprotection and promote the degeneration and apoptosis of testicular cells. The removal of one side of the GFN, i.e. the removal of the afferent nerve, can alleviate the damage to the contralateral testis. The present results therefore showed that the surgery of cryptorchidism or the removal of part of the GFN in the early clinical stages could help protect the contralateral testis from being further damaged.

The nerve conduction mechanism underlying the contralateral testicular damage in unilateral cryptorchidism is a complex process. Questions regarding whether other neurotransmitters besides CGRP are also involved, and whether exogenous CGRP has a protective effect on the contralateral testis, warrant further study.

\section{References}

1. Kumar V, Misro MM and Datta K: Simultaneous accumulation of hyaluronan binding protein 1 (HABP1/p32/gC1qR) and apoptotic induction of germ cells in cryptorchid testis. J Androl 33: 114-121, 2012.

2. Kollin C and Ritzén EM: Cryptorchidism: a clinical perspective. Pediatr Endocrinol Rev 11 Suppl 2: 240-250, 2014.

3. Lee PA, Bellinger MF, Songer NJ, O'Leary L, Fishbough R and LaPorte R: An epidemiologic study of patemity after cryptorchidism; intial results. Eur J Pediatr 152 Suppl 2: S25-S27, 1993.

4. Huff DS, Fenig DM and Canning DA: Abnormal germ cell development in cryptorchidism. Horm Res 55: 11-17, 2001.

5. Foresta C, Ferlin A, Garolla A, Milani C, Oliva G and Rossato M: Functional and cytologic features of the contralateral testis in cryptorchidism. Fertil Steril 66: 624-629, 1996.

6. Vasquez JM, Ben-Num L, Greenblatt RB, et al: Correlation between follicle-stimulating hormone, luteinizing hormone, prolactin, and testerone with sperm cell concentration and motility. Obstet Gynecol 67: 86-89, 1986.

7. Stewart RJ, Boyd S, Brown S and Toner PG: The blood-testis barrier in experiment unilateral cryptorchidism. J Pathol 160: $51-55,1990$.

8. Lenzi A, Geandini L, Lombardo F, et al: Unilateral cryptorchidism corrected in prepubertal age: evaluation of sperm parameters, hormones, and antisperm antibodies in adult age. Fertil Steril 67: 943-948, 1997.

9. Zempoalteca R, Martínez-Gómez M, Hudson R, Cruz Y and Lucio RA: An anatomical and electrophysiological study of the genitofemoral nerve and some of its targets in the male rat. J Anat 201: 493-505, 2002.

10. Kar S, Gibson SJ and Polak JM: Origins and projections of peptide-immunoreactive nerves in the male rat genitofemoral nerve. Brain Res 512: 229-237, 1990.

11. Patkowski D, Czernik KJ and Jelen M: Division of the genitofemoral nerve in unilateral cryptorchid rats. J Pediatr Surg 29: 832-835, 1994.

12. Morris HR, Panico M, Etienne T et al: Isolation and characterization of human calcitonin gene-related peptide. Nature 308: 746-748, 1984

13. Rasmussen TN, Bersani M, Schmidt P, et al: Isolation and molecular characterization of porcine calcitonin gene-related peptide (GGRP) and its endocrine effects in the porcine pancreas. Pancreas 16: 195-204, 1998.

14. Mimaki Y, Kawasaki H, Okazaki M, et al:Involvement of calcitonin gene-related peptide (CGRP) receptors in insulin-induced vasodilatation in mesenteric resistance blood vessels of rats. Br J Pharmacol 123: 1684-1690, 1998.

15. Benemei S, Nicoletti P, Capone JA and Geppetti P: Pain pharmacology in migraine: Focus on CGRP and CGRP receptors. Neurol Sci 28: S89-S93, 2007.

16. Ren YS, Ma TG, Wang and Yu SQ: Membrane fluidity changes in myocardial cells following severe hypoxia and simulated reperfusion and effects of calcitonin gene-related peptide. Med Sci Res 21: 627-628, 1993. 